\title{
Electron beam distortions in beam-beam compensation setup
}

\author{
Vladimir Shiltsev and Alexander Zinchenko* \\ Fermi National Accelerator Laboratory, Batavia, Illinois 60510
}

(Received 8 May 1998; published 6 October 1998)

\begin{abstract}
This article is devoted to electron beam distortions in the "electron compressor" setup for beambeam compensation in the Tevatron collider. The effects of electron space charge force and the interaction of the electron beam with the impacting elliptical antiproton beam are studied. We make an estimate of the longitudinal magnetic field necessary to keep the electron beam distortions low. [S1098-4402(98)00018-4]
\end{abstract}

PACS numbers: 41.20.-q, 41.75.Fr, 41.85.Ct, 29.27.Fh

\section{INTRODUCTION}

Proton and antiproton beams in the Tevatron collider interact via their electromagnetic forces at two collision points B0 and D0 and at numerous locations along separated orbits in the same vacuum chamber where they near miss each other. Such an interaction causes betatron oscillation tune shift and the tune spread in both beams. The tune shift and the tune spread are supposedly much larger in the antiproton beam than in the proton beam, because of several times larger proton intensity, and can reach values of about $0.01-0.02$ in the Tevatron luminosity upgrade project TEV33 [1]. These effects are expected to be a problem for the machine operation if uncorrected. Compensation of the beam-beam effects in the Tevatron with the use of high current, low energy electron beam was proposed in Refs. [2,3]. The electron beam travels in the direction opposite to the antiproton beam and interacts with an antiproton bunch via its space charge forces. The proton beam has to be separated from the electron and antiproton beams. Modifications of the proposal are (i) the "electron lens" with modulated current which is supposed to provide different linear defocusing forces for different antiproton bunches (the bunch spacing is $\tau=132 \mathrm{~ns}$ in the TEV33) and, therefore, equalize their betatron frequencies, which are not naturally equal due to proton-antiproton interaction in numerous parasitic crossings along the ring; and (ii) an "electron compressor," that is a nonlinear but DC electron lens which compensates (on average) the nonlinear focusing of antiprotons due to proton beam and, thus, to reduce the beam-beam footprint.

A $10 \mathrm{kV}$ electron beam about $2 \mathrm{~m}$ long, $2 \mathrm{~mm}$ diameter, with 1-2 A of current is to be installed in a place with large beta function $(\sim 100 \mathrm{~m})$, away from the main interaction points. The electron beam is to be born on an electron gun cathode, transported through the interaction region, and absorbed in the collector. A

\footnotetext{
*Permanent address: Joint Institute for Nuclear Research, 141980 Dubna, Moscow Region, Russia.
}

strong longitudinal magnetic field plays a significant role in maintaining stability of both electron and antiproton beams [4]. It also suppresses the electron beam current distribution distortions and, therefore, the electron space charge force distortions.

This article is focused mostly on the time-dependent deviations of the electron beam shape during passage of the interaction region. In Sec. II we discuss the results of numerical tracing of electron trajectories in the electron compressor. Theoretical analysis of the distortions in drift approximation is presented in Sec. III. Section IV briefly summarizes our studies.

\section{NUMERICAL TRACING OF ELECTRONS}

The ZBEAM code [5] is used for tracing electron trajectories. This is essentially a two-dimensional code which takes into account only transverse components of the electric and magnetic forces. It is a good approximation for the forces due to ultrarelativistic $\bar{p}$ bunch. Longitudinal space charge forces of the nonrelativistic electron beam are negligible because the beam is either DC or modulated over a large scale of $\tau \cdot c \approx 40 \mathrm{~m}$, which is much larger than the beam transverse size of a few $\mathrm{mm}$.

The code solves an equation of motion of a charge. In the laboratory frame, with some external electric and magnetic fields and in the presence of some additional moving electric charges, the equation is as follows:

$$
m \frac{d^{2} \vec{r}}{d t^{2}}=q\left(\vec{E}+[\vec{v} \times \vec{B}]+\sum_{i=1}^{n} \vec{E}_{i}+\sum_{i=1}^{n}\left[\vec{v} \times \vec{B}_{i}\right]\right),
$$

Here $m, q$, and $\vec{v}$ are the particle mass, electric charge, and velocity, $\vec{E}$ and $\vec{B}$ are the external electric and magnetic fields, and $\vec{E}_{i}$ and $\vec{B}_{i}$ are the electric and magnetic fields of a bunch "macroparticle"

$$
\vec{E}_{i}=\frac{q_{i}(Z) \vec{r}}{2 \pi \epsilon_{0} r^{2}}, \quad \vec{B}_{i}=\frac{1}{c^{2}}\left[\vec{v}_{i} \times \vec{E}_{i}\right] .
$$

Tracking of a particle is achieved by integrating the equation of motion over successive small time steps.

C 1998 The American Physical Society

064001-1 

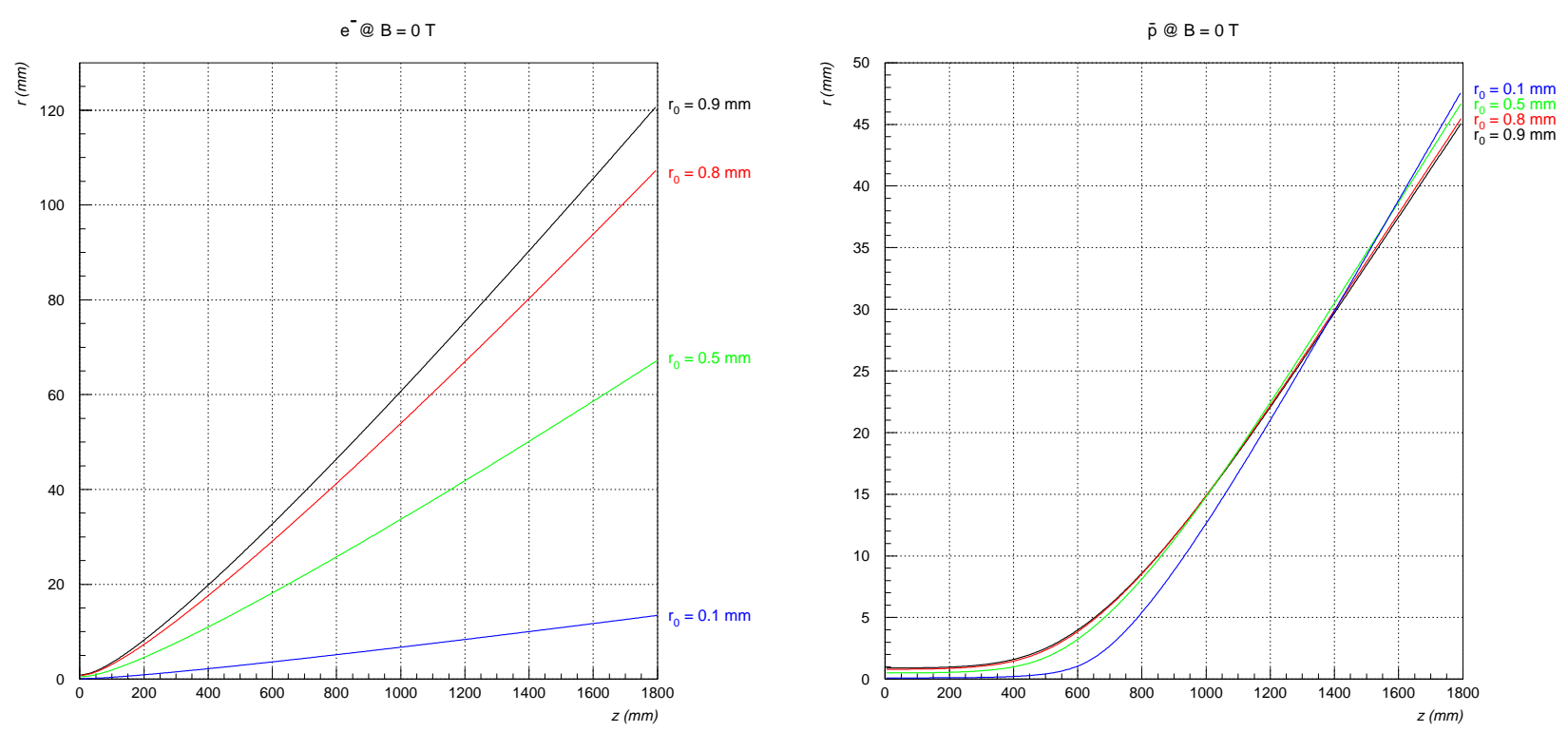

FIG. 1. (Color) Expansion of the electron beam due to self-field (left) and trajectories of electrons inside $\bar{p}$ bunch (right).

Figure 1 shows trajectories vs longitudinal coordinate $z$ for electrons which originally had no transverse velocities and started at radii equal to $r_{0}=0.1,0.5,0.8$, and $0.9 \mathrm{~mm}$ in the absence of longitudinal magnetic field.

The left plot demonstrates disruption under the impact of the self-space charge forces in a round electron beam with constant transverse current distribution with the following parameters: the beam radius $a=0.9 \mathrm{~mm}$, total current $J_{e}=1.5 \mathrm{~A}$, kinetic energy of electrons $U_{e}=$ $10 \mathrm{kV}$. One can see a many fold increase of the beam size over a $180-\mathrm{cm}$-long path. Note that the electron trajectories do not intersect each other; therefore, the particle at the border $r_{0}=0.9 \mathrm{~mm}$ always stays at the border.

The right plot in Fig. 1 shows trajectories of the same particles under the impact of the oncoming bunch of $N_{\bar{p}}=6 \times 10^{10}$ antiprotons having Gaussian distributions with rms radial size of $\sigma_{r}=0.9 \mathrm{~mm}$ and longitudinal rms size of $\sigma_{z}=30 \mathrm{~cm}$, and the electron space charge is off. Again, a significant electron beam size increase is seen; nevertheless, it is somewhat less than that due to electron space charge-final radius of about $50 \mathrm{~mm}$ instead of $120 \mathrm{~mm}$ - compared with the left plot.

Note that the $1800 \mathrm{~mm}$ path corresponds to the time for all antiprotons within $\pm 3 \sigma_{z}$ to add their impact to the electrons' motion. Now the trajectories intersect each other; thus the particle originally at the border of the electron beam received the least angular deflection.

The solenoid magnetic field in the setup for the beambeam compensation allows the avoidance of the disruption of the high current electron beam. As shown in Ref. [2], the electron beam stability in a solenoidal field $B$ requires its focusing strength to be more than defocusing due to electron and antiproton space-charge defocusing

$$
\frac{1}{F_{B}^{2}} \geq \frac{1}{F_{e}^{2}}+\frac{1}{F_{\bar{p}}^{2}},
$$

where the effective focal length due to the magnetic field $B$ is

$$
F_{B}=\frac{2 \gamma_{e} \beta_{e} m_{e} c^{2}}{e B} \approx 3.3[\mathrm{~cm}] \frac{\gamma_{e} \beta_{e}}{B[\mathrm{kG}]} .
$$

Here $\beta_{e}=v_{e} / c=\sqrt{2 U_{e} / m c^{2}}$ and $\gamma_{e}=1 / \sqrt{1-\beta_{e}^{2}}$ are relativistic factors; e.g., for $10 \mathrm{kV}$ electrons $\beta_{e}=0.2$ and

$$
F_{B}=0.66[\mathrm{~cm}] / B[\mathrm{kG}] .
$$

The defocusing length due to the electron space charge of the $1.5 \mathrm{~A}, 10 \mathrm{kV}$ electron beam is

$$
\begin{gathered}
F_{e}=\sqrt{\frac{J_{0} \gamma_{e}^{3} \beta_{e}^{3} a_{e}^{2}}{2 J_{e}}} \approx 0.77[\mathrm{~cm}], \\
J_{0}=\frac{m c^{3}}{e}=17 \mathrm{kA} .
\end{gathered}
$$

The minimum defocusing length due to the pbar beam is

$$
F_{\bar{p}}=\sqrt{\frac{\gamma_{e} \beta_{e}^{2} \sqrt{2 \pi} \sigma_{z} \sigma_{r}^{2} m_{e} c^{2}}{e^{2} N_{\bar{p}}\left(1+\beta_{e}\right)}} \approx 1.11[\mathrm{~cm}]
$$

where we take the same parameters as above $-N_{\bar{p}}=$ $6 \times 10^{10}, \sigma_{r}=0.9 \mathrm{~mm}, \sigma_{z}=30 \mathrm{~cm}$.

The electron beam is stable if the focusing term $1 / F_{B}^{2}$ in Eq. (3) is stronger than the two defocusing terms that corresponds to $B \geq 1 \mathrm{kG}$ for nonrelativistic electrons. The defocusing due to the electron space charge is about 1.5 times stronger than the defocusing due to the pbar beam; therefore, an approximate scaling law is valid for 
the minimum stabilizing solenoid field

$$
B_{\min } \propto \frac{J_{e}^{1 / 2}}{a_{e}}, \quad a_{e} \simeq \sigma_{r} .
$$

For example, doubling the electron current requires only $\sqrt{2} \approx 1.41$ more magnetic field strength.

Figure 2 presents electron trajectories in the $B=$ $2 \mathrm{~T}$ solenoidal magnet while taking into account the impact of both the electron and antiproton space charge forces. The electrons are assumed to be brought to the interaction region adiabatically, i.e., without excitation of their transverse Larmor oscillations with a spatial period of $\lambda_{L}=v_{e} / \omega_{L}=\beta_{e} m c^{2} / e B \approx 3.4[\mathrm{~mm}] / B[\mathrm{kG}]$. The $\bar{p}$ bunch length is much longer than $\lambda_{L}$, and, therefore, antiprotons repulse electrons adiabatically and do not excite the Larmor oscillations - one can see no radius variations in the top plot of Fig. 2.

The only effect of the space charge forces is an azimuthal drift of electrons as it is presented in the lower plot of Fig. 2. One can see that all electron trajectories started with a $y$ coordinate equal to 0 , but during the passage time all of the particles have been rotated while staying on the same radii. The drift velocity in the crossed electric and magnetic fields $\vec{E}$ and $\vec{B}$, respectively, is equal to

$$
\vec{v}_{d}=c \frac{[\vec{E} \times \vec{B}]}{B^{2}} .
$$

The space charge electric field inside the constant current density $j=J_{e} / \pi a_{e}^{2}$ is proportional to the radius $\vec{E}=2 j \vec{r} / \beta_{e}$, and, therefore, the angle $\theta_{d}$ of the drift rotation over the time interval $t$ does not depend on the radius $\theta_{d}=v_{d} t / r=2 j c t / \beta_{e} B$. The electric field due to the Gaussian $\bar{p}$ beam is not linear; therefore, the rotation angle $\theta_{d}$ is no longer independent of $r$, and electrons with larger $r$ perform drift rotation on a different (smaller) angle even though the difference is negligible under the parameters we used-see the lower plot in Fig. $2{ }^{1}$

One can conclude that the interaction with a round $\bar{p}$ bunch in the strong magnetic field conserves the axial symmetry and radial size of the electron beam. Therefore, the electron beam space charge forces are the same for antiprotons at the head and at the tail of the $\bar{p}$ bunch.

That is no longer true if the electron or the antiproton beam is not round. Roundness of the electron beam can be assured by using a round cathode in the electron gun

\footnotetext{
${ }^{1}$ In fact, the magnetic forces produced by the electron and antiproton currents produce additional drifts similar to electric ones, but their contributions are $\beta_{e}^{2}$ and $\beta_{e}$ times smaller and, therefore, are negligible. Nevertheless, it will be taken into account in the formulas of Sec. III.
}
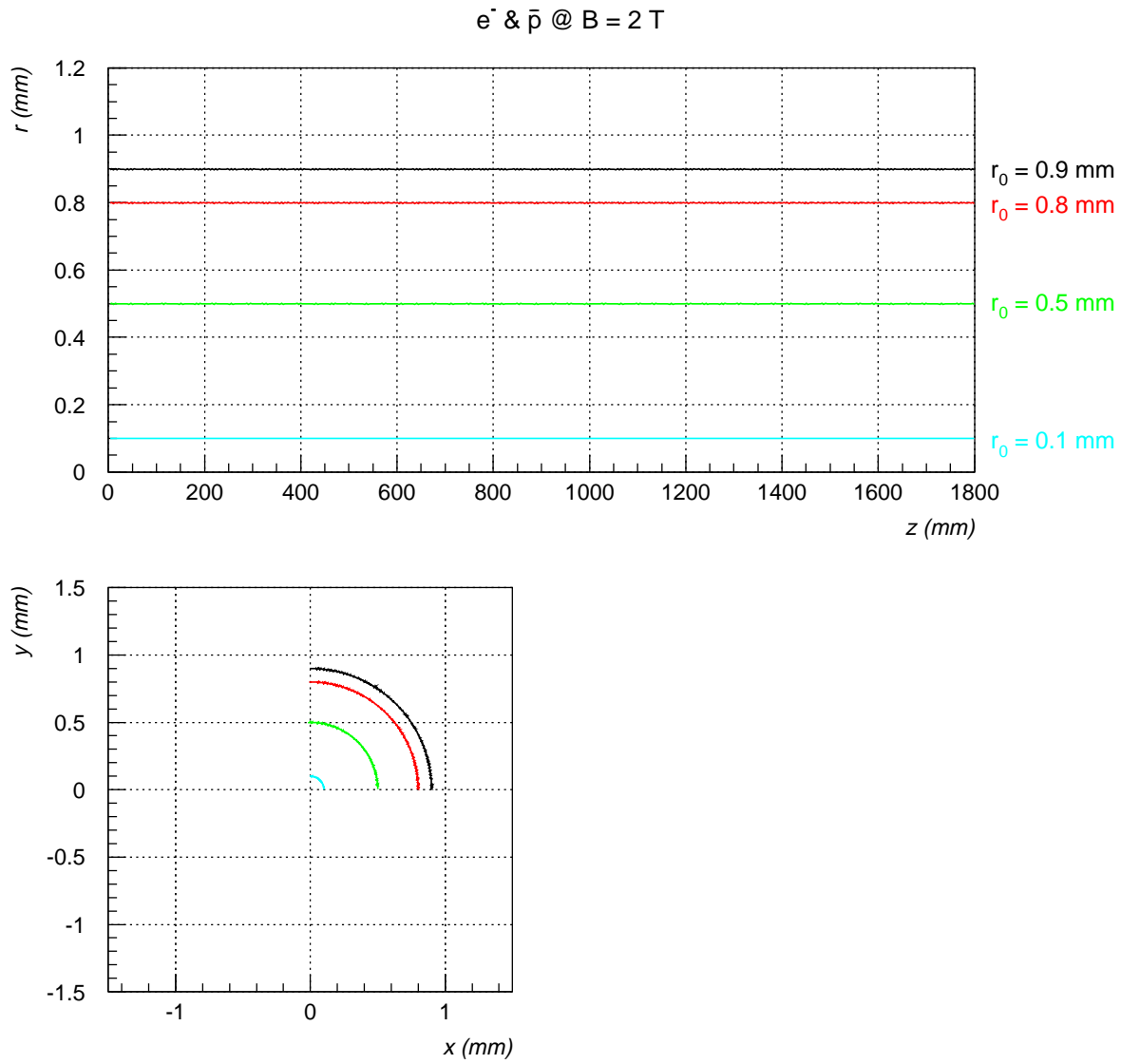

FIG. 2. (Color) Electron beam behavior inside $\bar{p}$ bunch at $2 \mathrm{~T}$. 
and by the appropriate choice of the magnetic field in the transport section of the setup. Oppositely, the $\bar{p}$ beam roundness can be achieved in very few Tevatron locations where vertical and horizontal beta functions are the same $\beta_{x}=\beta_{y}$ (vertical and horizontal emittances of $1000 \mathrm{GeV}$ beams in the Tevatron are approximately equal $\varepsilon_{x, y}^{\mathrm{rms}} \approx$ $3.3 \pi \mathrm{mm} \mathrm{mrad})$. This condition cannot be fulfilled a priori. For example, at the present stage we consider the installation of one of the electron lens devices at the Tevatron F48 location which is characterized by $\beta_{x}=$ $101.7 \mathrm{~m}$ and $\beta_{y}=30.9 \mathrm{~m}$, and, consequently, the rms bunch sizes are $\sigma_{x}=0.61 \mathrm{~mm}$ and $\sigma_{y}=0.31 \mathrm{~mm} \mathrm{[3].}$

Figure 3 shows what happens with a round electron beam (radius $a_{e}=0.31 \mathrm{~mm}$ ) when it interacts with elliptical antiproton beam in the $2 \mathrm{~T}$ solenoid field. The ZBEAM code is used for the electric field calculations and electron tracing. The top left plot shows an ellipse which corresponds to $1 \sigma$ of the Gaussian $\bar{p}$ bunch and a circle of the electron beam cross section uniformly filled with electron macroparticles before interaction with antiprotons. The top right plot demonstrates traces of the electrons under the impact of the asymmetric electric field of the antiproton bunch. The resulting macroparticle positions and the shape of the electron beam in $x-y$ and $r$ - $\phi$ planes are shown in the lower left and right plots of Fig. 3, respectively. One can see that the electron beam becomes a rotated ellipse at the moment when the tail of the antiproton bunch passes through it, while the head of the bunch sees the originally undisturbed round electron beam. This might be of concern for two reasons: (i) there appears a "head-tail" interaction in the $\bar{p}$ bunch via higher than dipole wake fields propagating in the electron beam, and (ii) in addition to the useful defocusing effect, electric fields of the elliptic electron beam produce effective $x-y$ coupling of the vertical and horizontal betatron oscillations in the $\bar{p}$ beam.

In the following section we analyze the effect and consider ways to reduce the distortion.

\section{ANALYSIS OF ELLIPTIC DISTORTIONS}

\section{A. Distortion of electron density}

We start with the continuity equation for the electron charge density $\rho(x, y, z, t)$

$$
\frac{\partial \rho}{\partial t}+\operatorname{div}(\rho \vec{v})=0
$$

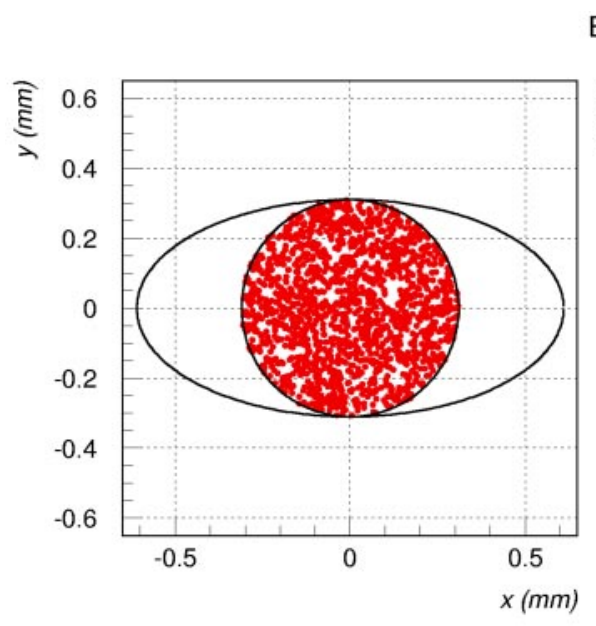

$$
B=2 T
$$
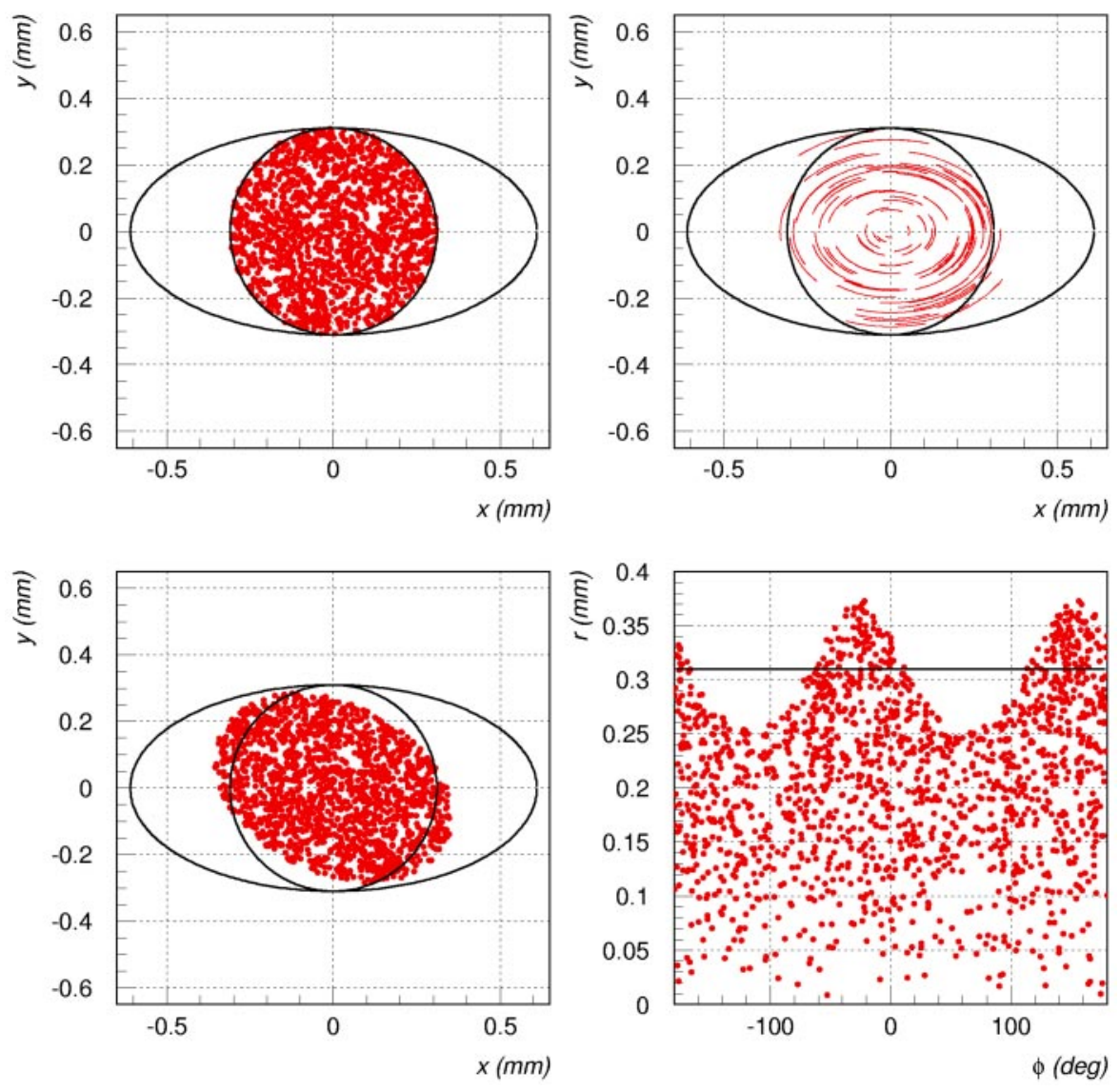

FIG. 3. (Color) Narrow electron beam distortion due to $\bar{p}$ bunch. 
where $\vec{v}(x, y, z, t)$ is the velocity of the electrons. Since the longitudinal component of the velocity is constant $v_{z}=\beta_{e} c$ and all of the longitudinal scales (such as the rms antiproton bunch length $\sigma_{z}$ or the electron beam length) are much longer than transverse scale, one can neglect the term $\partial / \partial z\left(\rho v_{z}\right)$ in (9). In the previous section, we found that the major component of the transverse electron motion is the drift with velocity $\vec{v}_{d}$ from Eq. (8), while the fast Larmor motion is negligible; therefore, in the further analysis we consider $\vec{v}=\vec{v}_{d}$. Now, if we assume that the unperturbed charge distribution is axially symmetric $\rho(t=0)=\rho_{0}(r)$ and that the maximum density distortion is small $\rho=\rho_{0}+$ $\delta \rho, \delta \rho \ll \rho_{0}$, in the lowest order one gets from (9)

$$
\frac{\partial \rho}{\partial t}+\vec{v}_{d} \cdot \vec{\nabla} \rho_{0}+\rho \operatorname{div} \vec{v}_{d}=0 .
$$

The third term is equal to zero because $\operatorname{div} \vec{v}_{d}=$ 0 . The gradient in the second term is $\vec{\nabla} \rho_{0}=$ $2 \vec{r} d^{2} \rho_{0}\left(r^{2}\right) / d\left(r^{2}\right)$; thus we obtain

$$
\vec{v}_{d} \cdot \vec{\nabla} \rho_{0}=\frac{2 c}{B^{2}} \frac{d \rho_{0}\left(r^{2}\right)}{d\left(r^{2}\right)}[\vec{E} \times \vec{B}] \cdot \vec{r} .
$$

The electric field of the round electron beam does not contribute to the product above as it is proportional to $\vec{r}$. Its contribution in our case can be omitted in further analysis as long as the electron charge density distortions are small with respect to $\rho_{0}(r)$. The major reason for the density change $\delta \rho$ is the antiproton beam space charge force. The electric field of the elliptic Gaussian relativistic $\bar{p}$ beam is given by

$$
\vec{E}=-e N_{\bar{p}} \lambda(z) \vec{\nabla} U,
$$

where the linear density of antiprotons is normalized as $\int \lambda(z) d z=1$, and the two-dimensional interaction potential $U(x, y)$ is [6]

$$
\begin{gathered}
U(x, y)=\int_{0}^{\infty} d q \frac{1-e^{-\left[x^{2} / 2 \sigma_{x}^{2}(1+q R)\right]-\left[y^{2} / 2 \sigma_{y}^{2}(1+q / R)\right]}}{\sqrt{(1+q R)(1+q / R)}}, \\
R=\frac{\sigma_{y}}{\sigma_{x}} .
\end{gathered}
$$

Therefore, after some mathematics, we get

$$
\begin{aligned}
\delta \rho\left[x, y, t=\frac{z}{\left(1+\beta_{e}\right) c}\right]= & {\left[\int_{-\infty}^{z} \lambda\left(z^{\prime}\right) d z^{\prime}\right] } \\
& \times \frac{2 e N_{\bar{p}}}{B} \frac{d \rho_{0}\left(r^{2}\right)}{d\left(r^{2}\right)} \\
& \times \frac{x y I(x, y)\left(\sigma_{x}^{2}-\sigma_{y}^{2}\right)}{\sigma_{x}^{2} \sigma_{y}^{2}},
\end{aligned}
$$

where $z$ is now the coordinate inside the $\bar{p}$ bunch $^{2}$ and

\footnotetext{
${ }^{2}$ For example, $z=-\infty$ is for the bunch head, and $\int_{-\infty}^{z} \lambda\left(z^{\prime}\right) d z^{\prime}$ is proportional to the antiproton charge which passed through the given part of the electron beam.
}

$$
\begin{aligned}
& I(x, y)=\int_{0}^{\infty} d q \frac{e^{-\left[x^{2} / 2 \sigma_{x}^{2}(1+q R)\right]-\left[y^{2} / 2 \sigma_{y}^{2}(1+q / R)\right]}}{(1+q R)^{3 / 2}(1+q / R)^{3 / 2}}, \\
& I(0,0)=\frac{2 R}{(1+R)^{2}} .
\end{aligned}
$$

Now we can see the major features of the resulting distortion: (i) it is absent in the case of round $\bar{p}$ beam when $\sigma_{x}=\sigma_{y}$; (ii) it performs two variations over azimuth $\delta \rho \propto x y \propto \sin (2 \theta)$; (iii) it vanishes with the solenoid field $B$ increase or a decrease of the antiproton intensity $N_{\bar{p}}$; (iv) most of the distortion takes place at the radial edge of the electron beam and, since $d \rho_{0}\left(r^{2}\right) / d\left(r^{2}\right) \simeq \rho_{0}^{\max } / a_{e}^{2}$, the wider electron beam gets smaller density distortions during the interaction.

Finally, the scaling of the maximum distortion strength is

$$
\frac{\delta \rho^{\max }}{\rho_{0}^{\max }} \sim \frac{0.2 e N_{\bar{p}}}{a_{e}^{2} B} \approx \frac{0.6\left[N_{\bar{p}} /\left(6 \times 10^{10}\right)\right]}{a_{e}^{2}[\mathrm{~mm}] B[\mathrm{kG}]},
$$

and the value of 0.2 comes from the geometrical factor $\propto x y I(x, y)$. For example, the distortion is about $3 \%$ for a 1-mm-radius electron beam in a $B=20 \mathrm{kG}=2 \mathrm{~T}$ solenoid field. As soon as the elliptic distortion is excited, it starts the rotation drift in the crossed fields of the electron space charge and the solenoid field. Under the conditions of the Tevatron beam-beam compensation setup, the rotation is small. For example, one gets that over the time of the $\bar{p}$ bunch passage $\pm 2 \sigma_{z} / c=2 \mathrm{~ns}$, the angle in the field of $B=20 \mathrm{kG}$ is about $\theta_{d} \approx$ $4 j \sigma_{z} a_{e} / \beta_{e} B \approx 0.1 \mathrm{rad} \ll 1$. Therefore, one can omit the factor $\vec{v}_{d} \cdot \vec{\nabla} \delta \rho$ in Eq. (10).

Figure 4 presents the ZBEAM simulations of the constant density electron beam which is much wider than the $\bar{p}$ beam $a_{e}=1.5 \mathrm{~mm} \approx 2.5 \sigma_{x}$. Opposite to the case presented in Fig. 3, the electron beam distortions in the same field of $B=2 \mathrm{~T}$ are now very small $\simeq 2 \%$.

The distortion of other than constant electron density can be calculated analytically with the use of Eq. (14). For example, the top left plot in Fig. 5 shows lines of constant density for the electron beam with a density of

$$
\begin{gathered}
\rho_{0}(r)=\frac{1}{1+\left(r / a_{e}\right)^{2 \mu}}, \\
\mu=3, \quad a_{e}=\sigma_{x}=0.61 \mathrm{~mm} .
\end{gathered}
$$

Here and below, the $x$ and $y$ coordinates are given in units of $\sigma_{x}$.

The constant density lines for the Gaussian distribution in the antiproton beam with $\sigma_{x}=0.61 \mathrm{~mm}$ and $\sigma_{y}=0.31 \mathrm{~mm}$ are presented in the top right plot. The lower left corner of the figure is for the change of the electron charge density $\delta \rho(x, y)$ after passage through the antiproton bunch with $N_{\bar{p}}=6 \times 10^{10}$ in the magnetic field $B=4 \mathrm{kG}$. With such a small solenoid field, the distortion is very large $\delta \rho^{\max } \simeq 0.25$, and the resulting electron beam shape $\rho=\rho_{0}+\delta \rho$ is now a rotated ellipse as it is 


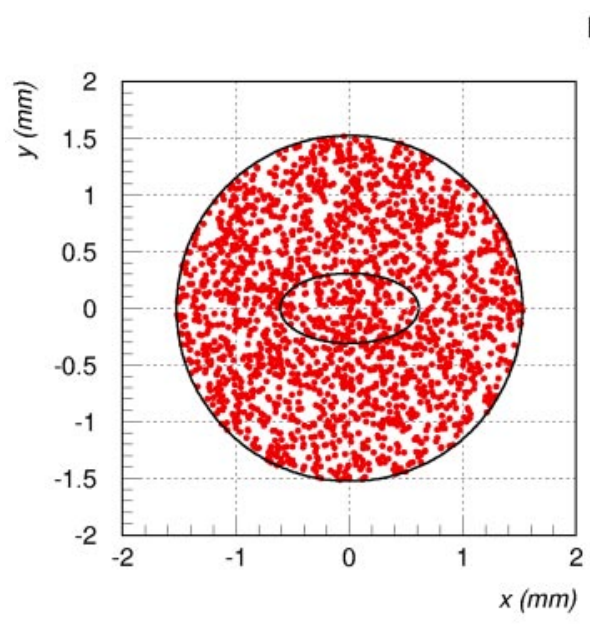

$$
B=2 T
$$
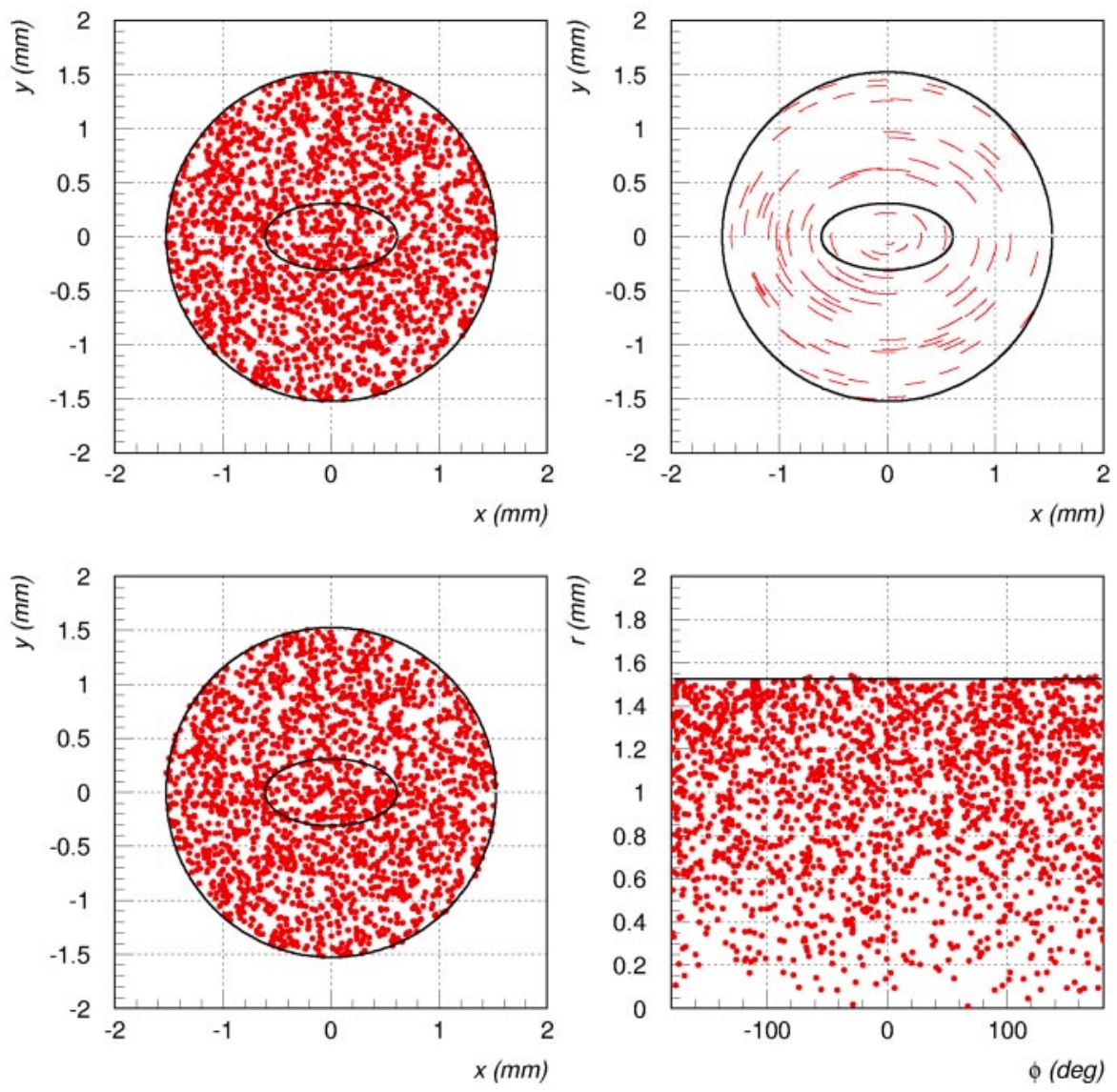

FIG. 4. (Color) Wide electron beam distortion due to narrow $\bar{p}$ bunch.

depicted in the lower right plot. Consequently, the kicks due to electron space charge fields are very different for the antiprotons in the head and in the tail of the bunch.

After consideration of the Tevatron beam-beam tune footprint in the presence of the electron lens in Ref. [3], it was found that an electron beam 2-3 times wider than the $\bar{p}$ beam size results in a smaller footprint area. That also helps to reduce $\delta \rho$. Figure 6 demonstrates the electron beam distribution $\rho_{0}$ according to Eq. (17), but with $a_{e}=$ $2.5 \sigma_{x}=1.5 \mathrm{~mm}$ (see left plot) and the resulting distortion $\delta \rho$ (right plot), which is now less than 0.05 .

\section{B. Coupling due to distorted electron beam}

The electric and magnetic fields of the elliptic electron beam lead to effective $x-y$ coupling of vertical and horizontal betatron oscillations in the $\bar{p}$ beam. Since the electron beam is originally round, the head of the $\bar{p}$ bunch experiences no coupling force. But, as the electron density distortion grows as $\int^{z} \lambda\left(z^{\prime}\right) d z^{\prime}$, the coupling grows proportionally. Particles in the head and in the tail of the bunch change their positions while performing synchrotron oscillations; thus an average coupling effect is half of the maximum coupling spread. The average coupling can be corrected in the Tevatron, while there are no tools to compensate the spread in the coupling. Therefore, the spread has to be small enough in order not to affect the $\bar{p}$ beam dynamics.

The tunes of a small amplitude particle can be written as

$$
\begin{aligned}
\nu_{ \pm}= & \frac{\left[\left(\nu_{x}+\Delta \nu_{x}\right)+\left(\nu_{y}+\Delta \nu_{y}\right)\right]}{2} \\
& \pm \sqrt{\frac{\left(\nu_{x}+\Delta \nu_{x}\right)-\left(\nu_{y}+\Delta \nu_{y}\right)^{2}}{4}+|\kappa+\Delta \kappa|^{2}},
\end{aligned}
$$

where $\nu_{x}$ and $\nu_{y}$ are the unperturbed horizontal and vertical tunes, respectively; in the current Tevatron lattice they are 0.585 and 0.575 , correspondingly. $\kappa$ is a complex number describing the coupling. For a satisfactory operation of the Tevatron collider, the global coupling is corrected down to a value of $|\kappa| \approx 0.001$ [7]. The $\Delta$ 's in Eq. (18) represent the changes of these quantities that arise from the interaction with the electron beam. The interaction is often described in terms of the twodimensional potential $V(x, y)$; thus the horizontal tune 

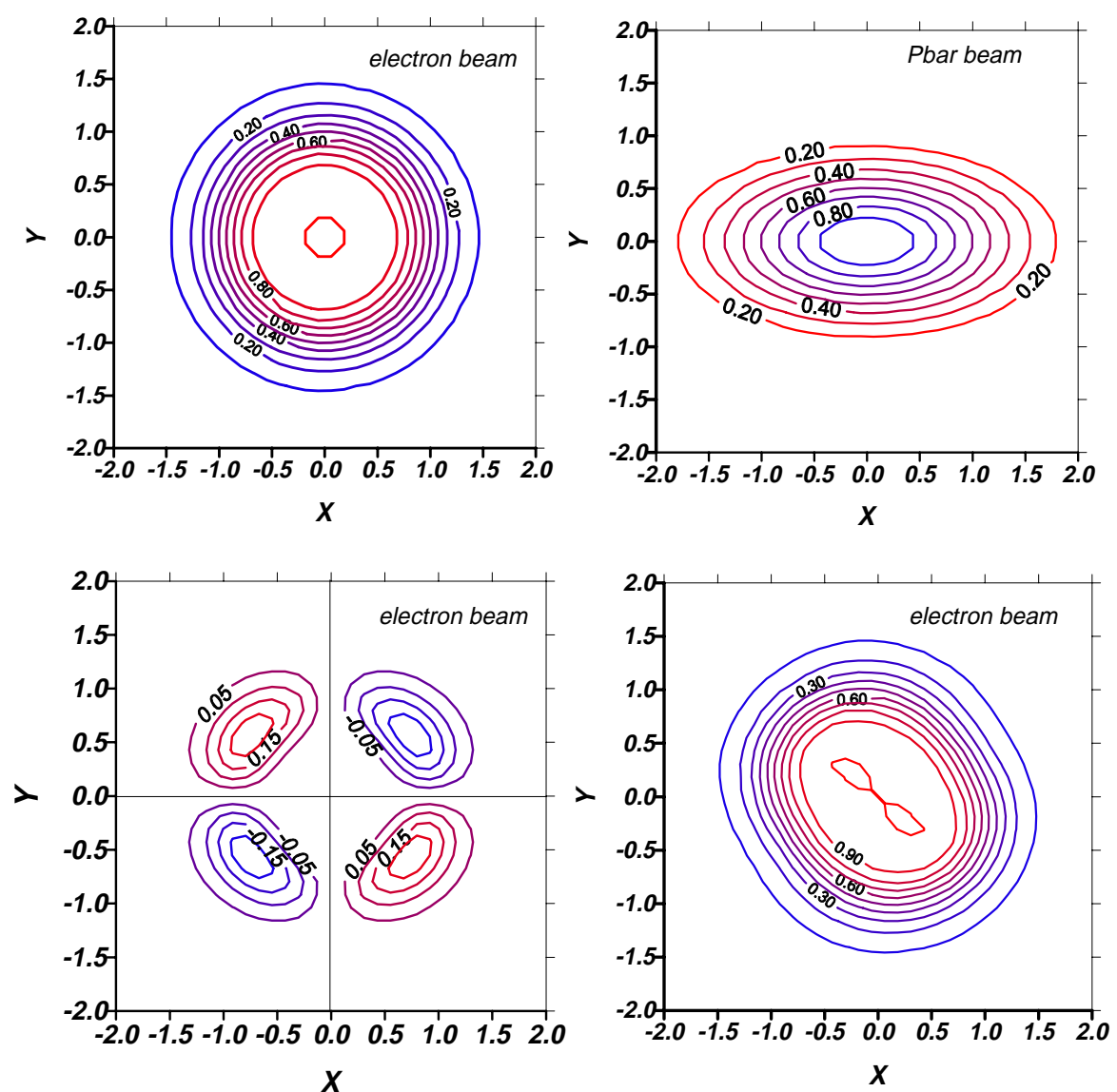

FIG. 5. (Color) Contour plots of the original electron density (top left), $\bar{p}$ density (top right), change of the electron density due to interaction with $\bar{p}$ space charge (bottom left), and the resulting electron density.

shift can be found from

$$
\Delta \nu_{x}=-\frac{\beta_{x}}{4 \pi} \frac{\partial^{2} V}{\partial x^{2}}
$$

The coupling shift can be calculated as

$$
\Delta \kappa=\frac{\sqrt{\beta_{x} \beta_{y}} e^{i\left(\psi_{x}-\psi_{y}\right)}}{4 \pi} \frac{\partial^{2} V}{\partial x \partial y} .
$$

In the case of the almost round electron beam with small elliptic distortion, one can write $V(x, y)=V_{0}(r)+$ $V_{\text {skew }}(x y)$. The potential $V_{0}(r)$ and corresponding tune shift for a round, constant density electron beam with total current $J_{e}$ and total length $L$ are equal to

$$
\begin{aligned}
V_{0}(r) & =r^{2} \frac{\left(1+\beta_{e}\right) J_{e} L r_{\bar{p}}}{e \beta_{e} c a_{e}^{2} \gamma_{\bar{p}}}, \\
\Delta \nu_{x} & =-\frac{\beta_{x}}{4 \pi} \frac{2\left(1+\beta_{e}\right) J_{e} L r_{\bar{p}}}{e \beta_{e} c a_{e}^{2} \gamma_{\bar{p}}} .
\end{aligned}
$$

Here $r_{\bar{p}}=1.53 \times 10^{-18} \mathrm{~m}$ is the (anti)proton classical radius, and the relativistic antiproton factor is $\gamma_{\bar{p}} \approx 1000$. For example, having parameters of experiment $J_{e}=$ $1.5 \mathrm{~A}, \beta_{e}=0.2, L=2.0 \mathrm{~m}, \beta_{x}=100 \mathrm{~m}$, and $a_{e}=$ $1 \mathrm{~mm}$, one gets $\Delta \nu_{x}=-0.0091$. The axisymmetric part of the electron beam distribution does not contribute in the coupling.

Now let us write the electron density distortion in the form $\delta \rho(x, y)=x y C(x, y)$, which emphasizes the product $x y$ and the rest is a slowly varying function of $x y$

$$
C(x, y)=\frac{2 e N_{\bar{p}}}{B} \frac{d \rho_{0}\left(r^{2}\right)}{d\left(r^{2}\right)} \frac{I(x, y)\left(\sigma_{x}^{2}-\sigma_{y}^{2}\right)}{\sigma_{x}^{2} \sigma_{y}^{2}} .
$$

The effective $2 \mathrm{D}$ skew potential can be found as a solution of the following equation:

$$
\triangle V_{\text {skew }}=-4 \pi \delta \rho \frac{r_{\bar{p}}}{\gamma_{\bar{p}}} .
$$

That is approximately equal to

$$
V_{\text {skew }} \approx \frac{\pi r_{\bar{p}}}{6 \gamma_{\bar{p}}} C(x, y) r^{2} x y .
$$

The magnitude of the coupling coefficient can be found with the use of Eq. (20)

$$
|\kappa| \approx \frac{\sqrt{\beta_{x} \beta_{y}} r_{\bar{p}}}{8 \gamma_{\bar{p}}}\left\langle C(x, y) r^{2}\right\rangle .
$$

The brackets $\langle\cdots\rangle$ denote averaging over antiproton betatron oscillations. Now one can estimate the maximum 

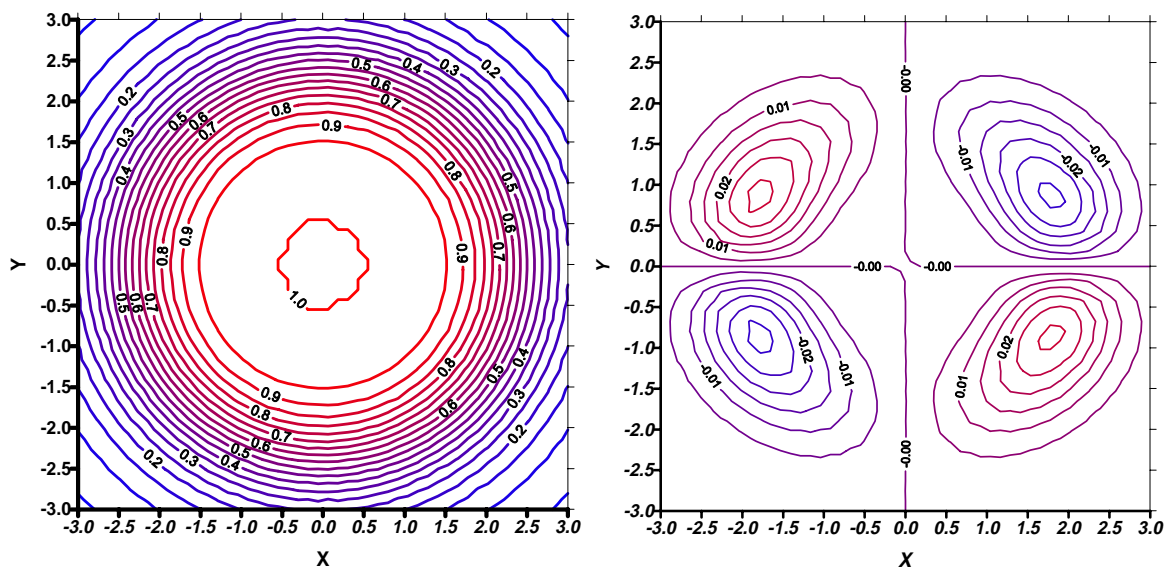

FIG. 6. (Color) Wider electron beam (left) and its distortion (right).

coupling spread using Eqs. (16) and (19)-(23) together with the approximate relation $\beta_{x} \simeq 3 \beta_{y}$

$$
\begin{aligned}
|\kappa| & \approx\left|\Delta \nu_{x}\right| \frac{e N_{\bar{p}}}{2 \sqrt{3} \sigma_{x}^{2} B}\langle S(x, y)\rangle \\
& \approx \frac{0.84\left[N_{\bar{p}} /\left(6 \times 10^{10}\right)\right]}{\sigma_{x}^{2}[\mathrm{~mm}] B[\mathrm{kG}]}\langle S(x, y)\rangle .
\end{aligned}
$$

Figure 7 shows the numerical factor $S(x, y)$ for the two electron distributions satisfying Eq. (17) with $a_{e}=\sigma_{x}$ (left plot) and another with $a_{e}=2.5 \sigma_{x}$ (right plot). The maximum value of this factor of $S^{\max }(x, y)=0.7$ for the slender electron beam and 0.13 for the wider electron beam takes place at amplitudes of about the electron beam size. The coupling vanishes for small betatron amplitude particles and at very large amplitudes. The effect is larger in the plane of the longer antiproton ellipse axis (horizontal in our case).

Let us assume a numerical example with the same parameters we used above $-\sigma_{x}=0.61 \mathrm{~mm}, N_{\bar{p}}=$ $6 \times 10^{10}, \Delta \nu_{x} \simeq 0.01$. The maximum numerical factor is about $\langle S(x, y)\rangle^{\max } \approx 0.5 S^{\max }(x, y)$; i.e., 0.35 for $a_{e}=$ $1 \sigma_{x}$ and 0.065 for $a_{e}=2.5 \sigma_{x}$. Now, with a solenoid field of $B=2 \mathrm{~T}$, one gets the maximum coupling spread $|\kappa| \simeq 4 \times 10^{-4}$ for the thin electron beam and $7 \times 10^{-5}$ for the wider electron beam. Both of these values are rather small with respect to the Tevatron global coupling correction goal of about 0.001 .

\section{CONCLUSION}

We have considered distortions of the electron beam in the beam-beam compensation setup. A rather low longitudinal field of about $1 \mathrm{kG}$ can prevent the beam blowup due to defocusing electron and antiproton space charge forces. A much higher solenoid field of about $2 \mathrm{~T}$ is necessary to have the electron charge distribution distortions within a few percent with respect to the original axisymmetric distribution. The need comes from a requirement to contribute much less $x-y$ coupling than other sources in the Tevatron collider ring and do not introduce significant spread of the coupling in the
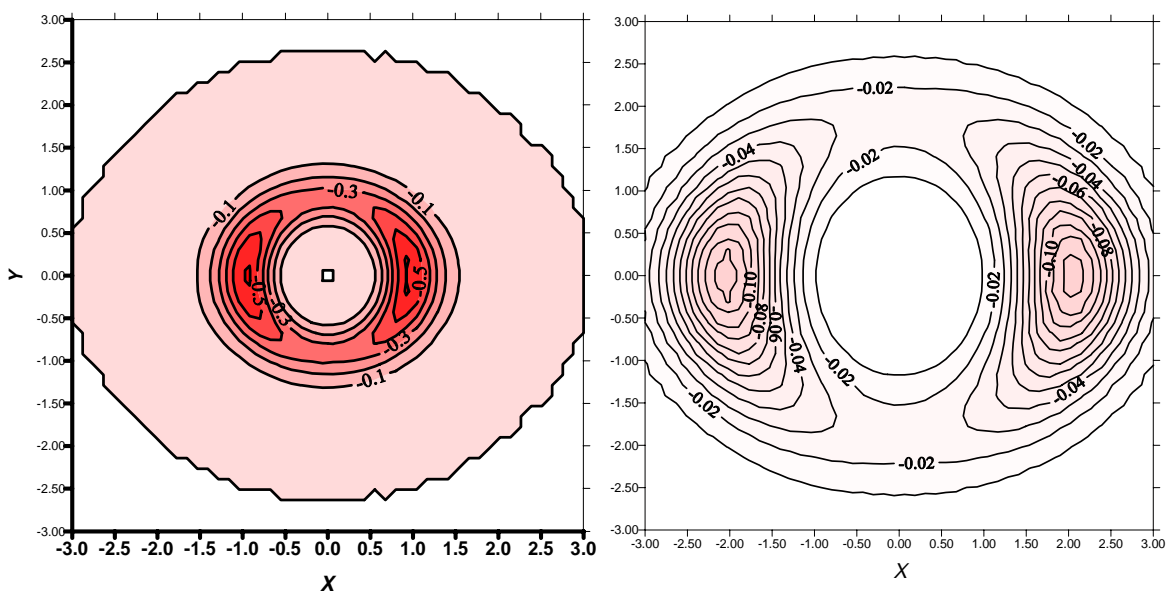

FIG. 7. (Color) The coupling functions $S(x, y)$ for antiproton betatron oscillations with thin (left) and wide electron beams (right). 
antiproton bunch. Both tracking with the computer code ZBEAM and analytical consideration have shown that the distortion is smaller if the electron beam size is several times the $\bar{p}$ beam size.

\section{ACKNOWLEDGMENT}

We acknowledge stimulating and fruitful discussions with Vasily Parkhomchuk, Slava Danilov, Andrei Sery, Peter Bagley, and Alexei Burov. We are thankful to Pat Smith for many corrections made after reading the manuscript.
[1] J.P. Marriner, Report No. FERMILAB-Conf-96/391, 1996; S. D. Holmes et al., Report No. FERMILAB-TM1920, 1995.

[2] V. Shiltsev and D. Finley, Report No. FERMILAB-TM2008, 1997.

[3] V. Shiltsev, Report No. FERMILAB-TM-2031, 1997.

[4] A. Burov, V. Danilov, and V. Shiltsev, Report No. FERMILAB-Pub-98/195, 1998; V. Shiltsev, Report No. FERMILAB-Conf-98/064, 1998.

[5] E. Tsyganov and A. Zinchenko, SSCL-Preprint-618, 1993.

[6] J. E. Augustin, SLAC Note PEP-63, 1973.

[7] P. Bagley and J. Annala (private communication). 